\title{
Vertical and horizontal variation of aerosol number size distribution in the boreal environment
}

Riikka Väänänen ${ }^{1}$, Radovan Krejci ${ }^{2,1}$, Hanna E. Manninen ${ }^{1}$, Antti Manninen ${ }^{1}$, Janne Lampilahti ${ }^{1}$, Stephany Buenrostro Mazon ${ }^{1}$, Tuomo Nieminen ${ }^{3,1}$, Taina Yli-Juuti ${ }^{3,1}$, Jenni Kontkanen ${ }^{1}$, Ari Asmi ${ }^{1}$, Pasi P. Aalto ${ }^{1}$, Petri Keronen ${ }^{1}$, Toivo Pohja ${ }^{1,4}$, Ewan O'Connor ${ }^{5}$, Veli-Matti Kerminen ${ }^{1}$, Tuukka Petäjä ${ }^{1}$, Markku Kulmala ${ }^{1}$

${ }^{1}$ Department of Physics, P.O. Box 64, FI-00014 University of Helsinki, Finland

${ }^{2}$ Stockholm University, Department of Environmental Science and Analytical Chemistry \& Bolin Centre for Climate Research, SE-10691 Stockholm, Sweden

${ }^{3}$ Department of Applied Physics, University of Eastern Finland, P.O. Box 1627, FI-70211 Kuopio, Finland

${ }^{4}$ SMEAR II measurement station, Hyytiäläntie 124, 35500 Korkeakoski, Finland

${ }^{5}$ Finnish Meteorological Institute, P.O. BOX 503, FI-00101 Helsinki, Finland

Correspondence to: R. Väänänen (riikka.vaananen@helsinki.fi)

\section{Supplementary material}

Table S1. The measurement flights done during the campaigns in May-June 2013 and March-April 2014. The days are classified based on the NPF events observed at the Smear II station. NPF event days are marked by 'E', and they are additionally divided to class 'EI' and 'EII' based on whether it was possible to calculate the formation rate and growth rate from the size distribution data, respectively. Class ' $N$ ' refers to non-event days, and class ' $U$ ' to undefined days. The case study flights are highlighted.

\begin{tabular}{|c|c|c|c|c|c|}
\hline $\begin{array}{l}\text { Flight } \\
\text { number }\end{array}$ & Date & Flight times & $\begin{array}{l}\text { Flight } \\
\text { around } \\
\text { Hyytiälä }\end{array}$ & $\begin{array}{l}\text { Day } \\
\text { class }\end{array}$ & $\begin{array}{l}\text { NPF } \\
\text { starting } \\
\text { time in } \\
\text { Hyytiälä }\end{array}$ \\
\hline 2013_1 & \multirow{2}{*}{24.04 .2013} & $14: 26-15: 28$ & - & \multirow{2}{*}{ EII } & \\
\hline 2013_2 & & $16: 40-17: 47$ & - & & \\
\hline 2013_3 & 29.04 .2013 & $13: 19-14: 45$ & - & EII & \\
\hline 2013_4 & 03.05 .2013 & 08:16-10:42 & $\mathrm{x}$ & EII & \\
\hline 2013_5 & \multirow[t]{2}{*}{06.05 .2013} & 08:04-10:44 & $\mathrm{x}$ & EI & \multirow[t]{2}{*}{ 11:40 } \\
\hline 2013_6 & & $16: 58-17: 56$ & $\mathrm{x}$ & & \\
\hline 2013_7 & \multirow[t]{2}{*}{ 07.05.2013 } & $09: 43-12: 28$ & $\mathrm{x}$ & \multirow[t]{2}{*}{ EII } & \multirow[t]{2}{*}{$11: 50$} \\
\hline 2013_8 & & $15: 24-17: 56$ & $\mathrm{x}$ & & \\
\hline 2013_9 & \multirow[t]{2}{*}{08.05 .2013} & 09:00-12:04 & $\mathrm{x}$ & \multirow[t]{2}{*}{ EI } & \multirow[t]{2}{*}{ 09:20 } \\
\hline 2013_10 & & $14: 49-16: 59$ & $\mathrm{x}$ & & \\
\hline 2013_11 & \multirow[t]{2}{*}{11.05 .2013} & $09: 47-12: 49$ & - & \multirow[t]{2}{*}{ EII } & \multirow[t]{2}{*}{ 13:00 } \\
\hline 2013_12 & & $15: 10-17: 12$ & $\mathrm{x}$ & & \\
\hline 2013_13 & \multirow{2}{*}{$\begin{array}{l}14.05 .2013 \\
15.05 .2013\end{array}$} & $10: 28-12: 58$ & $\mathrm{x}$ & \multirow{2}{*}{ EII } & \multirow[b]{2}{*}{ 13:20 } \\
\hline 2013_14 & & 09:27-12:12 & $\mathrm{x}$ & & \\
\hline $2013 \_15$ & \multirow[t]{2}{*}{16.05 .2013} & 08:11-10:52 & $\mathrm{x}$ & \multirow[t]{2}{*}{ EII } & \multirow[t]{2}{*}{ 09:00 } \\
\hline 2013_16 & & $13: 53-16: 22$ & $\mathrm{x}$ & & \\
\hline $2013 \_17$ & 17.05 .2013 & $09: 10-11: 45$ & $\mathrm{x}$ & $\mathrm{N}$ & \\
\hline
\end{tabular}




\begin{tabular}{|c|c|c|c|c|c|}
\hline 2013_18 & 18.05 .2013 & 09:10-11:58 & $\mathrm{x}$ & $\mathrm{U}$ & \\
\hline 2013_19 & 20.05 .2013 & $19: 06-21: 57$ & $\mathrm{x}$ & $\mathrm{U}$ & \\
\hline 2013_20 & 21.05 .2013 & 09:14-12:02 & $\mathrm{x}$ & $\mathrm{U}$ & \\
\hline 2013_21 & 22.05 .2013 & 09:30-12:07 & $\mathrm{x}$ & EII & $10: 10$ \\
\hline 2013_22 & & $14: 04-16: 14$ & $\mathrm{x}$ & & \\
\hline 2013_23 & 23.05 .2013 & 08:15-11:06 & $\mathrm{x}$ & EII & $08: 15$ \\
\hline 2013_24 & & $13: 42-16: 30$ & $\mathrm{x}$ & & \\
\hline $2013 \_25$ & 25.05 .2013 & $08: 43-11: 33$ & $\mathrm{x}$ & EII & $07: 45$ \\
\hline $2013 \_26$ & 26.05 .2013 & 06:53-09:52 & $\mathrm{x}$ & $\mathrm{U}$ & \\
\hline 2013_27 & & $12: 17-14: 46$ & $\mathrm{x}$ & & \\
\hline 2013_28 & 28.05 .2013 & 09:16-11:52 & $\mathrm{x}$ & $\mathrm{N}$ & \\
\hline 2013_29 & 29.05 .2013 & $10: 17-13: 17$ & $\mathrm{x}$ & $\mathrm{U}$ & \\
\hline 2013_30 & 31.05 .2013 & 06:49-09:31 & - & $\mathrm{U}$ & \\
\hline 2013_31 & 02.06 .2013 & 09:01-10:59 & $\mathrm{x}$ & $\mathrm{U}$ & \\
\hline 2013_32 & & $14: 21-16: 40$ & $\mathrm{x}$ & & \\
\hline 2013_33 & 03.06.2013 & $06: 40-09: 46$ & $\mathrm{x}$ & $\mathrm{U}$ & \\
\hline 2013_34 & & $12: 04-13: 12$ & $\mathrm{x}$ & & \\
\hline 2013_35 & 05.06 .2013 & $14: 44-16: 11$ & $\mathrm{x}$ & $\mathrm{U}$ & \\
\hline 2013_36 & 06.06 .2013 & 08:02-10:53 & - & $\mathrm{U}$ & \\
\hline 2013_37 & & $12: 52-15: 13$ & $\mathrm{x}$ & & \\
\hline 2013_38 & 08.06 .2013 & 07:43-10:41 & - & EII & $13: 30$ \\
\hline 2013_39 & & 21:04-23:59 & - & & \\
\hline 2013_40 & 09.06.2013 & $11: 52-13: 52$ & $\mathrm{x}$ & EII & $08: 50$ \\
\hline 2013 _41 & 10.05 .2013 & 06:15-09:00 & - & $\mathrm{U}$ & \\
\hline 2013_42 & & $11: 00-13: 03$ & - & & \\
\hline 2013_43 & 13.06.2013 & 07:50-10:43 & $\mathrm{x}$ & EII & 09:00 \\
\hline 2013_44 & & $12: 30-14: 59$ & $\mathrm{x}$ & & \\
\hline $2013 \_45$ & 15.06 .2013 & $09: 25-11: 43$ & $\mathrm{x}$ & EI & 09:00 \\
\hline 2014_1 & 24.04 .2014 & $17: 27-17: 59$ & - & EI & $12: 30$ \\
\hline 2014_2 & 25.03 .2014 & 09:04-11:23 & $\mathrm{x}$ & EI & $14: 00$ \\
\hline 2014_3 & & $14: 45-16: 51$ & $\mathrm{x}$ & & \\
\hline 2014_4 & 26.03 .2014 & $10: 16-12: 39$ & $\mathrm{x}$ & EI & $11: 30$ \\
\hline 2014_5 & & $15: 01-17: 24$ & $\mathrm{x}$ & & \\
\hline 2014_6 & 27.03 .2014 & $10: 03-12: 21$ & $\mathrm{x}$ & EI & 09:20 \\
\hline 2014_7 & & $15: 02-17: 09$ & $\mathrm{x}$ & & \\
\hline $2014 \_8$ & 28.03.2014 & $09: 52-12: 23$ & $\mathrm{x}$ & EI & $10: 40$ \\
\hline 2014_9 & & $14: 27-16: 30$ & $\mathrm{x}$ & & \\
\hline 2014_10 & 31.03 .2014 & 09:01-11:03 & $\mathrm{x}$ & EI & $12: 20$ \\
\hline 2014_11 & & $12: 46-14: 42$ & $\mathrm{x}$ & & \\
\hline 2014_12 & 01.04.2014 & 08:56-10:46 & $\mathrm{x}$ & EI & $15: 40$ \\
\hline 2014_13 & & $12: 37-14: 33$ & $\mathrm{x}$ & & \\
\hline 2014_14 & 02.04.2014 & 09:32-11:58 & $\mathrm{x}$ & EII & 09:30 \\
\hline
\end{tabular}




\begin{tabular}{|c|c|c|c|c|c|}
\hline 2014_15 & \multirow{3}{*}{03.04 .2014} & $14: 55-17: 21$ & $\mathrm{x}$ & \multirow{3}{*}{ EII } & \multirow{3}{*}{$10: 00$} \\
\hline 2014_16 & & 09:46-11:46 & $\mathrm{x}$ & & \\
\hline 2014_17 & & $13: 23-15: 41$ & $\mathrm{x}$ & & \\
\hline 2014_18 & 04.04 .2014 & $03: 27-05: 21$ & $\mathrm{x}$ & EI & $10: 15$ \\
\hline 2014_19 & \multirow{4}{*}{$\begin{array}{l}07.04 .2014 \\
08.04 .2014\end{array}$} & 09:30-11:39 & $x$ & & \multirow{4}{*}{ 09:20 } \\
\hline 2014_20 & & 09:38-11:52 & $\mathrm{x}$ & $\mathrm{U}$ & \\
\hline 2014_21 & & $10: 06-12: 26$ & $\mathrm{x}$ & EI & \\
\hline 2014_22 & & $14: 47-16: 52$ & $\mathrm{x}$ & & \\
\hline 2014_23 & \multirow[t]{2}{*}{09.04 .2014} & 09:45-12:02 & $\mathrm{x}$ & \multirow[t]{2}{*}{ EI } & \multirow[t]{2}{*}{$10: 30$} \\
\hline 2014_24 & & $14: 29-16: 55$ & X & & \\
\hline 2014_25 & \multirow[t]{2}{*}{10.04 .2014} & 07:59-10:24 & $\mathrm{x}$ & \multirow[t]{2}{*}{ EI } & \multirow[t]{2}{*}{ 08:00 } \\
\hline 2014_26 & & $12: 26-14: 56$ & $\mathrm{x}$ & & \\
\hline
\end{tabular}


Table S2. Class I NPF event days. Comparison of particle concentration and diameter between Hyytiälä station and aircraft-borne measurements inside the PBL. 10 ${ }^{\text {th }}$ an 90the percentiles (P10 and P90) of the total number concentration ( $\mathrm{N}_{\text {tot }}$ ), concentration of particles with diameter $10-25 \mathrm{~nm}\left(\mathrm{~N}_{10-25}\right)$ and $80-400 \mathrm{~nm}\left(\mathrm{~N}_{80-400}\right)$ are presented. $D_{\mathrm{p}}(<25 \mathrm{~nm})$ is the mode peak diameter of sub-25 nm mode when sub-25 nm particles were observed in Hyytiälä.

\begin{tabular}{|c|c|c|c|c|c|c|c|c|c|}
\hline & & $\mathrm{N}_{\text {tot }}$ & & $\mathrm{N}_{10-25}$ & & $\mathrm{~N}_{80-400}$ & & $D_{p}(<25 r$ & \\
\hline Flight & Date & Hyytiälä & Cessna & Hyytiälä & Cessna & Hyytiälä & Cessna & Hyytiälä & Cessna \\
\hline $2013 \_8$ & 8.5 .2013 & $\begin{array}{l}1800- \\
2300\end{array}$ & $\begin{array}{l}1600- \\
4500\end{array}$ & $\begin{array}{l}300- \\
600\end{array}$ & $\begin{array}{l}400- \\
1400\end{array}$ & $\begin{array}{l}200- \\
200\end{array}$ & $\begin{array}{l}100- \\
200\end{array}$ & $4-10$ & $<10-35$ \\
\hline 2013_9 & 8.5.2013 & $\begin{array}{l}4200- \\
4900\end{array}$ & $\begin{array}{l}5400- \\
7100\end{array}$ & $\begin{array}{l}1100- \\
1400\end{array}$ & $\begin{array}{l}1300- \\
3000\end{array}$ & $\begin{array}{l}300- \\
300\end{array}$ & $\begin{array}{l}300- \\
300\end{array}$ & $>25 \mathrm{~nm}$ & $>25 \mathrm{~nm}$ \\
\hline 2013_15 & 15.5.2013 & $\begin{array}{l}1800- \\
2000\end{array}$ & $\begin{array}{l}1900- \\
2600\end{array}$ & $\begin{array}{l}200- \\
300\end{array}$ & $\begin{array}{l}200- \\
400\end{array}$ & $\begin{array}{l}500- \\
600\end{array}$ & $\begin{array}{l}400- \\
700\end{array}$ & $13-23$ & $<10-42$ \\
\hline 2013_45 & 15.6.2013 & $\begin{array}{l}2400- \\
4900\end{array}$ & $\begin{array}{l}2600- \\
9700\end{array}$ & $\begin{array}{l}700- \\
2800\end{array}$ & $\begin{array}{l}900- \\
4800\end{array}$ & $\begin{array}{l}200- \\
300\end{array}$ & $\begin{array}{l}200- \\
300\end{array}$ & $8-19$ & $<10-22$ \\
\hline 2014_4 & 26.3.2014 & $\begin{array}{l}900- \\
1500\end{array}$ & $\begin{array}{l}1400- \\
3500\end{array}$ & $\begin{array}{l}300- \\
400\end{array}$ & $\begin{array}{l}300- \\
1000\end{array}$ & $0-100$ & $0-100$ & $7-17$ & $<10-30$ \\
\hline 2014_5 & 26.3.2014 & $\begin{array}{l}3300- \\
4900\end{array}$ & $\begin{array}{l}4200- \\
5700\end{array}$ & $\begin{array}{l}2000- \\
3000\end{array}$ & $\begin{array}{l}3000- \\
4500\end{array}$ & $\begin{array}{l}100- \\
200\end{array}$ & $\begin{array}{l}100- \\
100\end{array}$ & $14-16$ & $11-16$ \\
\hline 2014_6 & 27.3.2014 & $\begin{array}{l}2500- \\
4200\end{array}$ & $\begin{array}{l}4000- \\
6700\end{array}$ & $\begin{array}{l}400- \\
500\end{array}$ & $\begin{array}{l}600- \\
1400\end{array}$ & $\begin{array}{l}200- \\
400\end{array}$ & $\begin{array}{l}200- \\
700\end{array}$ & $5-5$ & $<10-39$ \\
\hline 2014_7 & 27.3.2014 & $\begin{array}{l}2500- \\
5100\end{array}$ & $\begin{array}{l}2500- \\
7700\end{array}$ & $\begin{array}{l}400- \\
2500\end{array}$ & $\begin{array}{l}600- \\
5300\end{array}$ & $\begin{array}{l}300- \\
400\end{array}$ & $\begin{array}{l}200- \\
400\end{array}$ & $7-16$ & $<10-16$ \\
\hline 2014_8 & 28.3.2014 & $\begin{array}{l}900- \\
17300\end{array}$ & $\begin{array}{l}1600- \\
27500\end{array}$ & $\begin{array}{l}200- \\
7800\end{array}$ & $\begin{array}{l}300- \\
13500\end{array}$ & $\begin{array}{l}100- \\
200\end{array}$ & $\begin{array}{l}100- \\
300\end{array}$ & $8-14$ & $<10-26$ \\
\hline 2014_9 & 28.3.2014 & $\begin{array}{l}12300- \\
13300\end{array}$ & $\begin{array}{l}14600- \\
21300\end{array}$ & $\begin{array}{l}5200- \\
6700\end{array}$ & $\begin{array}{l}7000- \\
13400\end{array}$ & $\begin{array}{l}200- \\
200\end{array}$ & $\begin{array}{l}200- \\
400\end{array}$ & $24-25$ & $11-25$ \\
\hline 2014_10 & 31.3.2014 & $\begin{array}{l}900- \\
1200\end{array}$ & $\begin{array}{l}1200- \\
2800\end{array}$ & $\begin{array}{l}300- \\
400\end{array}$ & $\begin{array}{l}400- \\
900\end{array}$ & $\begin{array}{l}100- \\
100\end{array}$ & $\begin{array}{l}100- \\
100\end{array}$ & $8-25$ & $11-27$ \\
\hline 2014_11 & 31.3.2014 & $\begin{array}{l}900- \\
1400\end{array}$ & $\begin{array}{l}900- \\
2100\end{array}$ & $\begin{array}{l}200- \\
300\end{array}$ & $\begin{array}{l}300- \\
500\end{array}$ & $\begin{array}{l}100- \\
100\end{array}$ & $\begin{array}{l}100- \\
100\end{array}$ & $8-10$ & $<10-26$ \\
\hline 2014_19 & 4.4.2014 & $\begin{array}{l}1700- \\
2000\end{array}$ & $\begin{array}{l}2100- \\
3800\end{array}$ & $\begin{array}{l}400- \\
600\end{array}$ & $\begin{array}{l}600- \\
1200\end{array}$ & $\begin{array}{l}100- \\
100\end{array}$ & $\begin{array}{l}100- \\
200\end{array}$ & $6-13$ & $<10-29$ \\
\hline 2014_21 & 8.4 .2014 & $\begin{array}{l}2700- \\
8700\end{array}$ & $\begin{array}{l}3400- \\
8300\end{array}$ & $\begin{array}{l}1000- \\
4800\end{array}$ & $\begin{array}{l}1200- \\
6500\end{array}$ & $\begin{array}{l}200- \\
300\end{array}$ & $\begin{array}{l}200- \\
300\end{array}$ & $9-21$ & $<10-22$ \\
\hline 2014_22 & 8.4.2014 & $\begin{array}{l}12200- \\
13300\end{array}$ & $\begin{array}{l}8300- \\
14900\end{array}$ & $\begin{array}{l}8200- \\
9800\end{array}$ & $\begin{array}{l}6200- \\
15000\end{array}$ & $\begin{array}{l}200- \\
200\end{array}$ & $\begin{array}{l}100- \\
200\end{array}$ & $13-16$ & $11-16$ \\
\hline 2014_23 & 9.4 .2014 & $\begin{array}{l}2400- \\
11600\end{array}$ & $\begin{array}{l}3400- \\
15000\end{array}$ & $\begin{array}{l}500- \\
1900\end{array}$ & $\begin{array}{l}600- \\
3300\end{array}$ & $\begin{array}{l}100- \\
200\end{array}$ & $\begin{array}{l}100- \\
200\end{array}$ & $5-8$ & $<10-24$ \\
\hline 2014_24 & 9.4 .2014 & $\begin{array}{l}10000- \\
12900\end{array}$ & $\begin{array}{l}10900- \\
16400\end{array}$ & $\begin{array}{l}5900- \\
6700\end{array}$ & $\begin{array}{l}7700- \\
15100\end{array}$ & $\begin{array}{l}200- \\
200\end{array}$ & $\begin{array}{l}200- \\
200\end{array}$ & $10-15$ & $<10-16$ \\
\hline 2014_25 & 10.4 .2014 & $\begin{array}{l}1800- \\
2300\end{array}$ & $\begin{array}{l}2100- \\
4000\end{array}$ & $\begin{array}{l}300- \\
600\end{array}$ & $\begin{array}{l}500- \\
1000\end{array}$ & $\begin{array}{l}400- \\
400\end{array}$ & $\begin{array}{l}400- \\
500\end{array}$ & $3-14$ & $17-30$ \\
\hline 2014_26 & 10.4 .2014 & $\begin{array}{l}6600- \\
10000\end{array}$ & $\begin{array}{l}11200- \\
17400\end{array}$ & $\begin{array}{l}500- \\
2000\end{array}$ & $\begin{array}{l}800- \\
7500\end{array}$ & $\begin{array}{l}400- \\
500\end{array}$ & $\begin{array}{l}400- \\
600\end{array}$ & $6-8$ & $<10-10$ \\
\hline
\end{tabular}

